\title{
On being second
}

\author{
Early adopters of new methods have a crucial role in validating them and defining their \\ limits. They deserve more formal recognition of this role.
}

Who does not want to be first? In virtually any field of study or physical endeavor, the first person to achieve the goal gets the accolades. Scientific research is no different and as a journal that aspires to publish the first definitive report of new and impactful biological research methods, Nature Methods is complicit in this.

But science progresses only when others reproduce and build on previous accomplishments and claims. If a new laboratory method could substantially improve the chances of obtaining new biological insights, a researcher may be sufficiently motivated to try it. But despite the best efforts of editors and reviewers to ensure that a manuscript is a reliable account of a method, adopting the method before others have documented that it works as advertised carries risks.

All too often, limitations or serious problems with methods do not emerge until after publication. Early adopters may have to expend considerable effort polishing a method or developing workarounds. On rare occasions they may run into a problem so fundamental that there is no solution. It is important that information about a problem that seriously affects a method is widely circulated to the community and linked from the original publication. Even if a high-profile retraction is unwarranted, communicating lesser problems - and preferably a solution-can save others from unnecessary grief.

We encourage early adopters who have encountered unexpected serious problems or limitations with methods we have published to communicate this through a Correspondence. This provides a transparent and peer-reviewed route for this information to reach the wider community, and gives early adopters a brief but formal high-profile acknowledgment of their contribution to a methodology.

The authors of the original report are always given an opportunity to respond to critiques and should do so without delay. We expect that authors of these critiques will have already discussed the problem with the original authors and that the documented problem is not a misunderstanding.

We occasionally receive a Correspondence critical of a specific paper published in another journal, but it is the responsibility of the original journal to consider these. If the method has been used in multiple publications in different journals, however, it is open to critique in a Nature Methods Correspondence.

Of course, Nature Methods is not the only venue for communicating and discussing problems with methods. Nature Protocols has a discussion forum suitable for this, and community forums such as SEQanswers. com and sharedproteomics.com can have a similar role. Open and searchable venues are always preferable to private exchanges in hallways and conferences.

Potential users of new methods must know the limitations before they can make informed decisions regarding the suitability of a method for their work. Some authors are refreshingly forthright about limitations of their new methods, and this does much to innoculate them from criticisms.

Although particular problems can be undetectable by the original developer and rely on early adopters to detect, some authors gloss over or omit known limitations while regaling readers with the capabilities of their wonderful new method. This is not only a disservice to the community but to the authors themselves. The limitations will become apparent; either during the review process or after publication. Our experience suggests that being upfront about known limitations at submission can improve the chances of publication.

Despite the potential benefits of using newly published methods before other researchers have had time to implement and validate them, these advantages may not improve the likelihood of a research grant relying on them being funded. In fact, it could have the opposite effect. The typical grant review process is an exercise in risk reduction, and proposing the use of such new methods, regardless of their potential, may hurt the chances of the grant being funded. Such a focus on the tried and true would be regrettable as it ignores the benefits to the community of trying new methods, even when they do not work as expected, and it inhibits an essential phase of methodological development.

The first manuscript describing a method or tool is only an early step on the road to establishing a method. The next steps taken by others are not as glamorous, but they are no less essential and can provide substantial rewards to those brave enough to take them. We thank early adopters for their role in validating and promulgating methods we have published and welcome their future contributions. 